\title{
Real Options Analysis for Commodity Based Mining Enterprises with Compound and Barrier Features
}

\author{
Otto Konstandatos (Corresponding author) \\ Discipline of Finance, The University of Technology, Sydney \\ P.O Box 123, Broadway, NSW, 2007 Australia \\ Tel: 61-2-9514-7758Ｅ-mail: otto..konstandatos@uts.edu.au \\ Timothy Kyng \\ Department of Actuarial Studies, Macquarie University, Sydney \\ Tel: 61-2-9850-7289 E-mail: timothy.kyng@mq.edu.au
}

Received: March 28, 2012

Accepted: November 6, 2012

Online Published: November 11, 2012

doi:10.5430/afr.v1n2p216

URL: http://dx.doi.org/10.5430/afr.v1n2p216

\begin{abstract}
Traditional project evaluations rely mainly on Net Present Value methodology, and largely ignore the flexibilities available to the sponsor to vary the project after initiation. Real Options Analysis remedies this by applying option pricing theory to more fully evaluate investment decisions. Through several hypothetical gold-mining examples, we illustrate the economic valuation of multi-stage investment decisions as simple or compound options, possibly with barrier option features. We present analytic valuation formulae for the types of compound options arising in this context, which differ from standard compound options. Barrier options are common in foreign exchange markets, and also arise in our analysis. We also present formulae for the valuation of the compound options appearing in our analysis with barrier features. It turns out that the decision to delay commencement contingent on commodity prices rising requires an up-and-in barrier option feature, whereas the risk of project nationalization may be modeled by adding an up-and-out barrier feature. Other barrier option features also arise in a Real Options context. We apply recently developed valuation methods for compound and barrier exotic options to several gold-mining examples, and we implement examples of the closed form valuation formulae using Excel spreadsheet software.
\end{abstract}

Keywords: Real options, NPV, Risk neutral valuation, Exotic options

\section{Introduction and Literature Review}

The concept of a real option was suggested by Myers (1977), who first identified many corporate economic assets as call or put 'real' options. Real options arise naturally where some economic decision needs to be made, as in manufacturing, production and other such situations. Some examples include: mine operators deciding what circumstances make it worthwhile to expand or alternatively abandon a mining operation; pharmaceutical firms making staged commitments to develop a new drug, from concept to research, trialing, legal approval, production and finally marketing. A real option is thus an option held by a corporation over some economic asset. It may be defined contractually or it may be more vaguely defined in terms of formal structure. The economic asset may be held or be under consideration for acquisition. Real options in the mining context arise naturally in the development of new plants, joint ventures and mineral exploration.

In this paper we consider several Real Option examples arising in the context of mineral exploration and mining projects. In particular we consider valuing such projects when the mine operator has the flexibility to defer project commencement, to expand the project or alternatively to abandon the project after commencement, or some combination. A project with an embedded option to defer which may also be expanded or contracted at a later date can be valued as a non standard type of compound option. We will also consider real options with barrier features and present closed form valuation formulae for several examples. We go on to consider incorporating barrier type features into these real options and discuss how it is plausible for the mining projects to have such barrier features.

Investment projects with the types of flexibility discussed above are typical of multi-stage investments, and they occur in mining, the pharmaceutical industry, real estate and IT. These have been extensively studied and attempts 
made to value such projects as compound options, which are options which have other options as the underlying assets. They may be referred to as 'options on options'. The first known valuation formulae for a European style compound option was that of Geske (1979), namely for an option to buy or sell a European Call or Put option, both of which are themselvesoptions to either buy or sell an underlying asset respectively. Compound options that arise in the analysis we present can be sequential with possibly several levels of compounding, making them more complex than those considered by Geske and hence more difficult to evaluate both analytically and numerically. The work of Hemantha et at (2002), Pendharker et al 2010 and Guj (2011) have explored the valuation of such sequential and compound options using numerical methods such as Monte Carlo Simulation and binomial lattices. The established closed-form option pricing formulae for standard compound and chooser options are not necessarily applicable to mining investment projects or to the examples considered in this paper.

Analytic formulae for the valuation of such non standard compound options and their corresponding barrier versions is obtained using the techniques and mathematical theory recently developed in the works of Buchen $(2001,2004)$, and Konstandatos $(2003,2008)$ which allow a systematic method for deriving analytic valuation formulae for the types of compound structures discussed in this paper using notions of static replication in terms of standardized instrument. The detailed discussion of these methods and theory is beyond the scope of this paper. Application of this approach was also undertaken in Kyng (2011) and Buchen, Konstandatos and Kyng (2008). Our valuation formulae are readily implemented using Excel/VBA or more sophisticated packages such as Matlab and Mathematica. Our purpose is to demonstrate how these non standard types of compound and barrier options arise naturally in a mining context, and to present the original valuation formulae along with some numerical worked examples. This fills a gap in the existing literature as analytic valuation formulae for these types of compound and barrier real options, along with the methods employed here to obtain them, are new and not well known.

This paper is structured as follows. Section 1 provides a brief introduction. Section 2 gives an overview of investment decision making and (standard) option pricing theory, and provides a context in which option pricing analysis arises naturally in the investment decision making process. Also provided is an overview of the non-standard methodology which we utilize to price the exotic options appearing in this paper. Section 3 contains the main contribution of this paper. In this section we analyze several investment decisions naturally arising in a hypothetical Gold-mining venture. These may include the flexibility to delay project commencement, the flexibility to abandon the project after commencement, the flexibility to expand production after commencement, or some combination. We demonstrate how such flexibilities are expressible as options with several exotic option features, and we provide closed-form formulae. Ultimately such flexibilities are seen to be equivalent to the introduction of compound and barrier option features in the 'real options' implicit in the project. We demonstrate their evaluation as exotic options over the underlying traded commodity, namely gold. Section 4 provides several numerically worked examples. Section 5 provides a brief conclusion.

\section{Real Option Analysis}

\subsection{Investment Decisions and Net Present Value}

The traditional investment decision rule is: invest only if the present value $(P V)$ of the expected future cashflows exceeds the cost of acquiring the asset. NPV is the present value of the asset's expected future cashflows less the cost of acquiring the asset. The traditional rule can be expressed as 'invest if the Net Present Value (NPV) of the project is positive': $N P V_{-}$now $>0 \Rightarrow$ invest. The NPV calculation uses risk adjusted discount rates to compute the present value of the expected future cashflows, and a 'best' cashflow estimate (guess). The risk adjusted discount rate is usually obtained via a financial economic model such as the well known capital asset pricing (CAPM) model. The NPV decision making rule has been recognized as flawed for some time. Dixit and Pindyk (1994) for example showed that it ignores the opportunity cost of making an immediate commitment, thereby giving up the option to wait for new information or better economic conditions. The opportunity cost can be thought of as the value of the option to defer the investment. A reformulation of the traditional investment decision making rule would thus be: 'invest now if $N P V_{\text {now }}>\max \left(N P V_{\text {defer }}, 0\right)$, where $N P V_{\text {defer }}$ is the value of the investment if postponed until some later time. Trigeorgis (1993) refers to this reformulation as the 'expanded NPV' rule, and provides several numerical examples of real options to defer, expand, contract and abandon.

\subsection{Risk Neutral Valuation for Real Options}

The assets underlying the options encountered in many kinds of real options analysis (ROA) are often not traded in a financial market. Black-Scholes option pricing theory (and indeed any theory postulating other asset price dynamics) depends on freely traded securities in liquid markets for the underlying asset. Nevertheless many leading researchers argue that it is valid to apply risk neutral valuation approaches to real options, and in particular the Black-Scholes 
framework. In his Nobel Prize lecture, Merton (1998) demonstrates that replication based valuation may still be used to price derivatives even when replication of the underlying security is not feasible because it is rarely traded. Arnold and Shockley (2002) demonstrate that the fundamental assumption of both the traditional NPV approach and the ROA approach is valuation by no arbitrage pricing principles. Trigeorgis (1993) implicitly assumes the Black-Scholes framework by numerically approximating the values of several examples via the Binomial pricing method. In our hypothetical gold mining examples however, we express the project flexibilities as options over the underlying gold price. As gold is actively traded in a highly liquid market, objections to applying option pricing theory due to an illiquid underlying asset are negated. This also holds for numerous mining examples we have not considered here.

\subsection{Compound and Barrier Option features}

Compound options are options to buy or sell another (underlying) option. Formulae for call and put compound options on European option underlying assets were developed by Geske (1979). Extensions include the work of Carr (1988) on the valuation of sequential exchange opportunities; the work of Buchen (2004) on the replication of dual expiry exotics in terms of standardized instruments; the work of Konstandatos $(2003,2008)$ which gives formulae and methodology to price more generalized compound options with both barrier and lookback option underlying assets and features; the work of Lee et al (2008) on generalised sequential compound options, and the work of Kyng (2011) on the application of exotic option pricing theory to the valuation of compound and barrier real options. The type of compound options we consider are more complex than the standard compound options, which may involve options to buy or sell a combination of a call (or put) option and a forward contract. We refer to these as non standard compound options. We demonstrate that generalized compound options arise naturally in a real options context, with the underlying asset not necessarily a standard European call or put. In this paper we value several hypothetical gold mining projects with embedded flexibilities as generalized compound options and their barrier option versions, and provide several numerical implementations in Excel spreadsheets.

Corporations are subject to resource constraints (capital rationing) and have alternative investment opportunities that compete for funding. Additionally, in certain circumstances governments and regulators may intervene to cancel or take over projects. During the 'waiting period' to proceed, it is also possible that project creditors (financiers) may cancel funding if the spot price of the commodity drops too low. We will demonstrate how this may be thought of as a down-and-out type barrier option. Alternatively, if the commodity price rises above some threshold, the sponsor might have the option to invest in another more profitable project, or the government may nationalize the project. Similarly, these possibilities give rise to up-and-out barrier options. Accordingly a real option to delay investment in a gold mine could involve up and out or down and out barrier option features. Barrier options over underlying equities contain provisions allowing effective cancellation if the price of the underlying asset (e.g. a commodity price) drops below (or rises above) some threshold price level $B$. In our context, this may represent some threshold for profitability. Alternatively, the option may come into existence when the commodity price rises above this profitability threshold.

\subsection{Review of Option Pricing Theory}

The Black-Scholes Option Pricing model, building on Samuelson (1965), was developed in the early 1970s and is now considered a classic result in the Finance industry. Using the idea of efficient markets, Black and Scholes (1973) demonstrated that an option over a stock has an economic value depending on $x$, (the market price of the stock) and $t$, (the time elapsed since the option was written). The model assumes the stock price process is geometric Brownian motion, and makes several idealized assumptions about market frictions. Let $V(x, t)$ be the value at time $t$ value of some option contract defined over an asset with current value $x . V(x, t)$ satisfies the Black-Scholes Partial Differential Equation (PDE) on the domain $D=\{(x, t) \mid x>0,0<t<T\}$, subject to the terminal boundary condition $V(x, t)=f(x)$ :

$$
\frac{\partial V}{\partial t}+(r-y) x \frac{\partial V}{\partial x}+\frac{1}{2} \sigma^{2} x^{2} \frac{\partial^{2} V}{\partial x^{2}}-r V=0
$$

The parameters $(r, y, \sigma, \tau)$ represent the risk free interest rate, the dividend yield, the volatility of the asset and the time to option maturity respectively. A European call of strike $K$ has payoff $f(x)=\max (x-K, 0)$ and satisfies $V(0, t)=0$, namely the option value is zero if the asset becomes worthless. The solution subject to the relevant boundary conditions for a European call is:

where

$$
\begin{gathered}
C(x, K, r, y, \sigma, \tau)=x e^{-y \tau} N\left(d_{1}\right)-K e^{-r \tau} N\left(d_{2}\right) \\
{\left[d_{1}, d_{2}\right]=\frac{1}{\sigma \sqrt{\tau}}\left[\ln \left(\frac{x}{K}\right)+\left(r-y \pm \frac{1}{2} \sigma^{2}\right) \tau\right]}
\end{gathered}
$$

PDE methods were first used to derive option pricing formulae. An alternative approach due to Harrison and Pliska (1981) obtains the option price by computing the expected option payoff under the equivalent martingale measure 
(also known as the risk neutral distribution), discounted at the risk free rate of interest. That this is mathematically equivalent to solving PDE (1) subject to the boundary conditions follows from a celebrated theorem of Feynman \& Kac (Kac (1949)).

Various numerical methods exist for option pricing, most notably Monte Carlo simulation (Boyle (1977)) and the binomial method (Cox, Ross and Rubinstein (1979)). The binomial method is a discrete time, discrete state space approximation which models the asset price distribution as "log-binomial" rather than log-normal. Another approach is to apply finite difference methods for parabolic PDEs to the Black-Scholes equation: Hull and White (1990). Numerical methods are typically applied when it is not possible to derive analytical valuation formulae.

\subsection{Barrier options and their pricing}

Barrier options are a class of path dependent options introduced in Merton (1973). The path dependence of barrier options is the simplest possible, consisting of a payoff dependent on whether the realised asset path reaches a certain barrier level. There are two major types of barrier contracts. The knock-in barrier option features are triggered if the asset reaches the barrier level, whereas the knock-out barrier option expires worthless if the barrier level is hit. The down and out barrier option $V_{D O}(x, t)$ satisfies PDE (1) and expiry condition $V_{D O}(x, T)=f(x)$, but with an extra boundary condition $V_{D O}(b, t)=0, t<T$ on the restricted domain $D=\{(x, t) \mid x>b, 0<t<T\}$. This option is 'cancelled' if the asset price crosses the level $B$ (the barrier level) from above before the option maturity date. Otherwise, the same payoff as the standard option (e.g. call or put) results.

Barrier options with a single, constant, knock-out boundary were first considered in Merton (1973). Given any payoff $f(x)$, four basic types of barrier option are possible. These are the down and out, the down and in, the up and out and the up and in barrier options. There are two fundamentally different ways to price barrier options, the 'standard' expectations approach (Rubinstein and Reiner (1991), Rich (1994)) and the PDE method. The expectations approach requires the determination of risk-neutral densities as the barrier level is breached by use of the reflection principle of Brownian Motion (see Harrison (1985)). On the other hand, the PDE approach (see Wilmott et al (1995)) involves solving the Black Scholes PDE subject to an extra boundary condition representing the knock-out or knock-in feature.

Our approach to barrier option pricing was pioneered in Buchen (2001). A full account of the methodology is provided in Konstandatos (2008) in the constant barriers case, including extensions of the theory to options with double barrier, lookback and complex compound and hybrid exotic option features. This approach was recently extended to exponential and time-varying barrier levels in Buchen and Konstandatos (2009). These authors demonstrate that prices for all four types of barrier options may ultimately be expressed as portfolios of European (path independent) options, considerably simplifying the explicit valuation of such instruments. The authors also give methods to deal with double barrier features. The basic approach is illustrated in the following.

Theorem [Image Solution]. Let $V_{B}(x, t)$ satisfy PDE (1) on domain $D=\{(x, t) \mid x>0,0<t<T\}$ with terminal boundary condition $V_{B}(x, T)=f(x) I_{(x>B)}$. Given any such solution, the 'image' function $V_{B}^{*}(x, t)$ with respect to the barrier level $x=B$ is another solution of PDE (1) defined by a transformation of dependent variable and scaling:

$$
V_{B}^{*}(x, t)=\left(\frac{B}{x}\right)^{\alpha} V_{B}\left(\frac{B^{2}}{x}, t\right) \text { where } \alpha=2 \frac{(r-y)}{\sigma^{2}}-1
$$

with terminal payoff $V_{B}^{*}(x, T)=\left(\frac{B}{x}\right)^{\alpha} f\left(\frac{B^{2}}{x}\right)$.

Clearly, the 'image option' as defined above is an abstract construction. However, Buchen (2001) derives the prices of the standard knock-out barrier call and put options by use of several image function properties, and provides symmetries for determining the knock-in option versions. The Buchen (2001) approach is readily extended to solve the general knock-out barrier problem as a simple difference (Konstandatos $(2003,2008))$ :

$$
V_{D O}(x, t)=V_{B}(x, t)-V_{B}^{*}(x, t),
$$

for any payoff $f(x)$. An equivalent result holds for the general up-and-out barrier option pricing problem. We thus convert the difficult problem with a knock-out barrier condition into a simpler European option problem for computing $V_{B}^{*}(x, t)$ either analytically (as we do in this paper) or numerically, from which the knock-out (whether $\mathrm{D} / \mathrm{O}$ or $\mathrm{U} / \mathrm{O}$ ) barrier option price follows. The corresponding knock-in barrier option prices are obtained by use of the symmetry properties of the image operation. Konstandatos (2008) gives details and mathematical proofs. Buchen and Konstandatos (2009) provides further details and extensions to time-varying and double-knock-out boundaries.

\section{Hypothetical Gold Mining Project Real Options}

Option pricing theory naturally arises when expressing the flexibility inherent in real managerial decisions. To motivate the discussion of barrier and compound barrier real options, we consider a hypothetical gold mine project. 
Gold is a mineral, a commodity and an investment asset actively traded on financial markets. The market price is readily observable, along with gold futures prices, and the prices of options on gold and other financial derivatives. We begin by considering a simple mining project without any options to expand or abandon, after possible project initiation at some future point in time. Later we include flexibilities to expand or abandon mining operations, making the valuation more complex.

\subsection{The basic project}

Suppose the project sponsor has the option to commence some Gold mining project at some future time $T_{0}$. By deciding to invest, the sponsor must outlay an initial amount of capital $K_{0}$. In return, profits are received at times $T_{1}, T_{2}, \ldots, T_{n}$ of amount $X_{T_{i}}-C$ at time $T_{i}$ respectively, where $X_{T_{i}}$ is the time $T_{i}$ market price of gold and $C$ is the cost of extracting and processing the gold each period. We assume the cost of extraction is constant, and that by committing the sponsor is 'locked' into the project and the cashflows. When using NPV methodology to decide whether to invest at time $T_{0}$, we compute the expected present value (PV) of the project's future cash flows:

$$
P V=e^{-r\left(T_{i}-T_{0}\right)} \sum_{i=1}^{n} E\left\{\left(X_{T_{i}}-C\right) \mid X_{T_{0}}\right\}
$$

Using arbitrage arguments, Hull (2009) demonstrates that the risk neutral expectation of the gold price at future time $T_{i}$ conditional on its time $T_{0}$ price equals the time $T_{0}$ forward commodity price at time $T_{0}$ for delivery at time $T_{i}$. The relationship between the forward price for delivery at time $T_{i}$ and the commodity price at time $T_{0}$ is $E\left\{X_{T_{i}} \mid X_{T_{0}}\right\}=X_{T_{0}} e^{-(r-q)\left(T_{i}-T_{0}\right)}$, which is a standard result from financial economics. The parameter $q$ is the income yield on the mined commodity, adjusted for storage costs, convenience yield and so on. It may be estimated from the prices of futures or forward contracts over the commodity.

Assuming that the extraction costs are uncorrelated with stock market returns, the capital asset pricing model (CAPM) allows us to discount these costs at the risk free rate of interest, namely:

$$
\begin{gathered}
\sum_{i=1}^{n} e^{-r\left(T_{i}-T_{0}\right)} E\left\{X_{T_{i}} \mid X_{T_{0}}\right\}=X_{T_{0}} \sum_{i=1}^{n} e^{-q\left(T_{i}-T_{0}\right)}=X_{T_{0}} A(q, 0, n) \\
\sum_{i=1}^{n} e^{-r\left(T_{i}-T_{0}\right)} E\{C\}=C \sum_{i=1}^{n} e^{-r\left(T_{i}-T_{0}\right)}=C A(r, 0, n)
\end{gathered}
$$

where $A(r, m, n)=\sum_{i=m+1}^{n} e^{-r\left(T_{i}-T_{m}\right)}$ is an annuity factor. Note that for $m<n$ we have

$$
A(r, 0, n)=\sum_{i=1}^{n} e^{-r\left(T_{i}-T_{m}\right)}=A(r, 0, m)+A(r, m, n) e^{-r\left(T_{m}-T_{0}\right)}
$$

The time $T_{0}$ value of the project's future cashflows is therefore a function of the $T_{0}$ gold price, namely $P V_{0}=$ $A(q, 0, n) X_{T_{0}}-C A(r, 0, n)$. The NPV of the project at time $T_{0}$ can be written as $N P V_{0}=\alpha_{0}\left(X_{T_{0}}-K_{0}^{\prime}\right)$, which is $\alpha_{0}=A(q, 0, n)$ units of a forward contract over gold with delivery price $K_{0}^{\prime}=\beta_{0}+\frac{K_{0}}{\alpha_{0}}$ and maturity date $T_{0}$, where $\beta_{0}=C \frac{A(r, 0, n)}{A(q, 0, n)}$.

\subsection{The option for future commencement}

Suppose an investor has the option to invest in a gold mine project at time $T_{0}$ for capital outlay $K_{0}$. The investor has the choice to delay the project start in the hope of more favourable market conditions. At time $T_{0}$ however, the sponsor is not obligated to commence operations if conditions remain unfavourable. Using NPV methodology, they proceed with the investment if the NPV is positive and don't proceed otherwise. The sponsor will exercise the right when $X_{T_{0}}>K_{0}^{\prime}$. Therefore the sponsor's option to 'delay' the commencement of the project and incur the relevant costs has time $T_{0}$ cashflow $\max \left(P V-K_{0}, 0\right)$, i.e. $\alpha_{0}\left[X_{T_{0}}-K_{0}^{\prime}\right]^{+}$. This is the payoff of $\alpha_{0}$ units of a call option over gold, with strike price $K_{0}^{\prime}=\beta_{0}+\frac{K_{0}}{\alpha_{0}}$. The project with an option for future commencement may thus be valued for $t<T_{0}$ using the Black-Scholes formula for a European call option with $T_{0}$ maturity and modified strike $K_{0}^{\prime}$.

3.3 The option to abandon the project

Assume the project sponsor has the right (but is not obligated) to permanently abandon the project at some future time $T_{m}<T_{n}$ after commencing at $T_{0}$, and to receive the mine's salvage value $S_{m}$ if so chosen. The value of the project's foregone cashflows at time $T_{m}$ is given by $P V_{m}=A(q, m, n) X_{T_{m}}-C A(r, m, n)$. The sponsor will abandon the project if the salvage value exceeds $P V_{m}$. The right to abandon can be written as an option payoff:

$$
\left(S_{m}+C A(r, m, n)-X_{T_{m}} A(q, m, n)\right)^{+}=A(q, m, n)\left(\frac{S_{m}+C A(r, m, n)}{A(q, m, n)}-X_{T_{m}}\right)^{+}
$$


where $(X)^{+}=\max (X, 0)$, so can be valued for times $t<T_{m}$ as $A(q, m, n)$ units of a European put option over gold, with maturity date $T_{m}$ and modified strike price $K_{m}=\frac{S_{m}+C A(r, m, n)}{A(q, m, n)}$.

At time $T_{m}$ the sponsor chooses between abandoning the project or continuing based on whether the salvage value exceeds the present value of the foregone cash-flows, namely covering project times $t \geq T_{m}$ they choose the $T_{m}$ cashflow:

$$
\begin{aligned}
& \max \left(S_{m}, X_{T_{m}} A(q, m, n)-C A(r, m, n)\right) \\
& \quad \equiv X_{T_{m}} A(q, m, n)-C A(r, m, n)+\left(S_{m}+C A(r, m, n)-X_{T_{m}} A(q, m, n)\right)^{+}
\end{aligned}
$$

Using put call parity between European call and put options, we can rewrite this as:

$$
S_{m}+\left(A(q, m, n) X_{T_{m}}-\left(C A(r, m, n)+S_{m}\right)\right)^{+}=S_{m}+A(q, m, n)\left(X_{T_{m}}-\frac{S_{m}+C A(r, m, n)}{A(q, m, n)}\right)^{+} .
$$

This is the salvage value plus the payoff from $\alpha_{m}=A(q, m, n)$ units of a European call option over gold with exercise price $K_{m}$. This has time $T_{0}$ value:

$$
S_{m} e^{-r\left(T_{m}-T_{0}\right)}+\alpha_{m} C\left(X_{T_{0}}, K_{m}, r, q, \sigma, T_{m}-T_{0}\right)
$$

where $C($ ) denotes the European call price as a function of the above arguments. We note that this is a monotonic increasing function of the gold price $X_{T_{0}}$. The $T_{0}$ value of the project's future cashflows including the right to abandon at future time $T_{m}>T_{0}$ is therefore

$$
P V_{0}=\alpha_{0}\left(X_{T_{0}}-\beta_{0}\right)+S_{m} e^{-r\left(T_{m}-T_{0}\right)}+\alpha_{m} C\left(X_{T_{0}}, K_{m}, r, q, \sigma, T_{m}-T_{0}\right)
$$

where $\alpha_{0}=A(q, 0, m) ; \beta_{0}=C \frac{A(r, 0, m)}{A(q, 0, m)} ; \alpha_{m}=A(q, m, n) ; \beta_{m}=C \frac{A(r, m, n)}{A(q, m, n)}$.

\subsection{The option to expand the project}

The project sponsor may have the option to expand the project at future time $T_{p}$. This could involve a new deposit of ore, with differences in remaining life, cost of extraction and initial cost. For instance it may be that the cost of extraction is higher at $\hat{C}$ per period, and the new project produces cashflows at times $T_{i}$ fori $i=p+1, p+2, \ldots, N$ where $N \neq n$ and the initial outlay required at time $T_{p}$ is of amount $K_{p}$. The time $T_{p}$ value of the project extension cashflows is

$$
P V_{T_{p}}=\hat{\alpha}_{p}\left(X_{T_{p}}-\hat{\beta}_{p}\right), \text { where } \hat{\alpha}_{p}=A(q, p, N) \text { and } \hat{\beta}_{p}=\widehat{C} \frac{A(r, p, N)}{A(q, p, n)} .
$$

Suppose at time $T_{p}$ the sponsor may expand by commencing this new project for cost $K_{p}$. The payoff from the option to expand is thus $\hat{\alpha}_{p}\left(X_{T_{p}}-\widehat{K}_{p}\right)^{+}$, namely $\hat{\alpha}_{p}$ units of a call option over gold, with exercise price $\widehat{K}_{p}=\frac{K_{p}}{\widehat{\alpha}_{p}}+\hat{\beta}_{p}$ and maturity $T_{p}$. The $T_{0}$ value of this option is:

\subsection{Flexible versions of the project}

$$
V_{0}^{E x p}=\hat{\alpha}_{p} C\left(X_{T_{0}}, \widehat{K}_{p}, r, q, \sigma, T_{p}-T_{0}\right)
$$

We now consider scenarios where a sponsor has several further 'flexible' project alternatives to vary the project down the track once commenced. Specifically, when commencing at time $T_{0}$ the sponsor may have the further right to either expand the project, to abandon the project or possibly do either at some future time. We therefore conceive of three versions of flexibility: the right to commence the project plus the right to abandon only (i.e. without the right to expand); the right to commence plus the right to expand only (without the right to abandon), and finally the right to commence plus the right to expand as well as the right to abandon. The option to expand is independent of the original project, but the option to abandon is contingent on the existence of the original project. We will outline the evaluation of the first version, and present the result for a barrier version of the project. Similar results exist for all three versions of the project which we omit here.

\subsubsection{Compound option evaluation}

In this section we evaluate a flexible version of the mining project which includes options to both abandon and expand. Assume it costs $K_{0}$ to enter into the project at initiation. The $T_{0}$ value of the flexible version of the project including the option to abandon at time $T_{m}>T_{0}$ as well as the option to expand at $T_{p}>T_{0}$ is given by:

$$
N P V_{T_{0}}\left(X_{T_{0}}\right)=\alpha_{0}\left(X_{T_{0}}-\beta_{0}\right)+S_{m} e^{-r\left(T_{m}-T_{0}\right)}-K_{0}+\alpha_{m} C\left(X_{T_{0}}, \widehat{K}_{m}, T_{m}-T_{0}\right)+\hat{\alpha}_{p} C\left(X_{T_{0}}, \widehat{K}_{p}, T_{p}-T_{0}\right)
$$


This includes the cashflows for times $t \leq T_{m}$, and consists of a long forward contract, a zero coupon bond and two long call options. Since call option prices are strictly increasing with respect to stock price, $N P V_{T_{0}}\left(X_{T_{0}}\right)$ is strictly increasing in argument $X_{T_{0}}$. Namely, there will be a unique value $X_{T_{0}}=a$ say for which $N_{P} V_{T_{0}}(a)=0$ and for which $N P V_{T_{0}}(x)>0 \Leftrightarrow x>a$. It follows that this flexible project may be valued for $t<T_{0}$ as an exotic compound option:

$$
V_{t}=e^{-r \tau_{0}} E\left\{\left[\alpha_{0}\left(X_{T_{0}}-\beta_{0}\right)+S_{m} e^{-r \tau_{m}}-K_{0}+\alpha_{m} C\left(X_{T_{0}}, \widehat{K}_{m}, \tau_{m}\right)+\hat{\alpha}_{p} C\left(X_{T_{0}}, \widehat{K}_{p}, \tau_{p}\right)\right] I_{(x>a)} \mid X_{T_{0}}=x\right\}
$$

which is decomposable into four expectations:

$$
\begin{aligned}
V_{t}=\alpha_{0} e^{-r \tau_{0}} E & \left\{X_{T_{0}} I_{\left(X_{T_{0}}>a\right)} \mid X_{T_{0}}=x\right\}+e^{-r \tau_{0}} E\left\{\left(S_{m} e^{-r \tau_{m}}-K_{0}-\alpha_{0} \beta_{0}\right) I_{\left(X_{T_{0}}>a\right)} \mid X_{T_{0}}=x\right\} \\
& +\alpha_{m} e^{-r \tau_{0}} E\left\{C\left(X_{T_{0}}, \widehat{K}_{m}, \tau_{p}\right) I_{\left(X_{T_{0}}>a\right)} \mid X_{T_{0}}=x\right\}+\widehat{\alpha}_{p} e^{-r \tau_{0}} E\left\{C\left(X_{T_{0}}, \widehat{K}_{p}, \tau_{p}\right) I_{\left(X_{T_{0}}>a\right)} \mid X_{T_{0}}\right. \\
& =x\}
\end{aligned}
$$

where $\tau_{m}=T_{m}-t, \tau_{p}=T_{p}-t, \tau_{0}=T_{0}-t$. The result may be expressed as a sum of four standardised dual expiry (namely, generalized compound) options of the type defined and used in Konstandatos (2003), Buchen (2004) and Konstandatos (2008). Defining the arguments

$$
d_{a}(x, \tau)=\frac{1}{\sigma \sqrt{\tau}}\left[\ln (x / a)+\left(r-q+\frac{1}{2} \sigma^{2}\right) \tau\right], d_{a}^{\prime}(x, \tau)=d_{a}(x, \tau)-\sigma \sqrt{\tau}
$$

the valuation formula $V(x, t)$ for the project at time $t$ and observed (market) gold price $x$ involves both the uni-variate and bi-variate normal distributions:

$$
\begin{aligned}
& V(x, t)=\alpha_{0} x e^{-q \tau_{0}} N\left(d_{a}\left(x, \tau_{0}\right)\right)+\alpha_{m} Q_{a, \widehat{K}_{m}}^{++}\left(x, \tau_{0}, \tau_{m}\right)+\hat{\alpha}_{p} Q_{a, \widehat{K}_{p}}^{++}\left(x, \tau_{0}, \tau_{p}\right) \\
& +e^{-r \tau_{0}}\left(S_{m} e^{-r\left(T_{m}-T_{0}\right)}-K_{0}-\alpha_{0} \beta_{0}\right) N\left(d_{a}^{\prime}\left(x, \tau_{0}\right)\right)
\end{aligned}
$$

where we have defined the following function for any $\left(x, a, K, \tau_{1}, \tau_{2}\right)$ :

$$
Q_{a, K}^{++}\left(x, \tau_{1}, \tau_{2}\right)=x e^{-q \tau_{2}} N_{2}\left(d_{a}\left(x, \tau_{1}\right), d_{K}\left(x, \tau_{2}\right) ; \sqrt{\frac{\tau_{1}}{\tau_{2}}}\right)-K e^{-r \tau_{2}} N_{2}\left(d_{a}^{\prime}\left(x, \tau_{1}\right), d_{K}^{\prime}\left(x, \tau_{2}\right) ; \sqrt{\frac{\tau_{1}}{\tau_{2}}}\right)
$$

The above function falls within the class of generalized dual-expiry exotic compound options defined in Buchen (2004). $N_{2}(x, y ; \rho)$ denotes the bi-variate normal CDF with standard Normal marginals and correlation $\rho$. We may add a knock-out barrier window in the vesting period before project initiation knocking the project out if the gold price sinks to some lower level deemed too uneconomic, $x=b$ say. Denoting $a^{\prime}=\max (a, b)$ and $x^{\prime}=b^{2} / x$, by application of the method of images, the version of the project with a knock-out (barrier) feature has price:

$$
\begin{aligned}
V(x, t)=\alpha_{0} x e^{-q \tau_{0}}\left(N\left(d_{a^{\prime}}\left(x, \tau_{0}\right)\right)-\left(\frac{b}{x}\right)^{\alpha} N\left(d_{a^{\prime}}\left(x^{\prime}, \tau_{0}\right)\right)\right)+\alpha_{m}\left(Q_{a^{\prime}, \widehat{K}_{m}}^{++}\left(x, \tau_{0}, \tau_{m}\right)-\left(\frac{b}{x}\right)^{\alpha} Q_{a^{\prime}, \widehat{K}_{m}}^{++}\left(x^{\prime}, \tau_{0}, \tau_{m}\right)\right) \\
+\hat{\alpha}_{p}\left(Q_{a^{\prime}, \widehat{K}_{p}}^{++}\left(x, \tau_{0}, \tau_{m}\right)-\left(\frac{b}{x}\right)^{\alpha} Q_{a^{\prime}, \widehat{K}_{p}}^{++}\left(x^{\prime}, \tau_{0}, \tau_{m}\right)\right) \\
+e^{-r \tau_{0}}\left(S_{m} e^{-r\left(T_{m}-T_{0}\right)}-K_{0}-\alpha_{0} \beta_{0}\right)\left(N\left(d_{a^{\prime}}^{\prime}\left(x, \tau_{0}\right)\right)-\left(\frac{b}{x}\right)^{\alpha} N\left(d_{a^{\prime}}^{\prime}\left(x^{\prime}, \tau_{0}\right)\right)\right)
\end{aligned}
$$

Similar expressions are obtained for the other versions of the flexible project described above. The above formulae are easily programmed and valuated in Excel/VBA or Matlab.

Options to delay a project may include further options to expand or to abandon in the future. It is conceivable that once a project is expanded, the expanded part of the project may itself include another option to expand, or alternatively to abandon the expansion, thus involving compound options on compound options and their barrier versions. Such scenarios naturally give rise to sequential compound options. Our approach is readily extended to price many such examples and their barrier versions not included here, with valuation formulae involving the cumulative bi-variate, tri-variate and higher dimensional Normal distribution functions.

\section{Numerical Examples}

For illustration, we present numerical valuations of several example projects and their associated real options. The calculations were implemented using Microsoft Excel spreadsheet software, which has many useful built-in functions and tools including the uni-variate cumulative Normal distribution. Calculations requiring the bi-variate normal cumulative density function were done using a VBA program implementation of Drezner's algorithm documented in Hull (2009).

Example 1: Assume a gold mining project with a three year life. Every three months we can extract 1,000 oz of gold and sell it into the market. The cost involved in producing the gold is $\$ 900.00$ per oz. The current gold price is $\$ 1000$ 
per oz. The risk free rate of interest is $8 \%$ p.a. and the current dividend yield on gold is $2 \%$ p.a. It will cost $\$ 2,000,000$ to commence the project. If we proceed immediately, the value of the project's future cashflows is $P V=\alpha_{0}\left(X_{T_{0}}-\beta_{0}\right)$ where $X_{T_{0}}=1000000, \alpha_{0}=11.870898, \beta_{0}=900000 \frac{10.562276}{11.870898}=800786$, giving a PV of 2364849. The NPV is $\max (P V-$ cost, 0$)=364849>0$, so we proceed with the investment.

Example 2: Next we consider the value of the option to wait before committing to invest, as we think it likely the gold price will move down rendering future operations less viable. Assume a gold price volatility of $20 \%$ p.a., with an option to wait for three months before committing to the investment. By the preceding analysis, the option to delay the decision to invest is a call option over gold with a term of three months, a gold 'spot price' of $\$ 1000 / \mathrm{oz}$, an exercise price of

$\widehat{K}_{0}=\beta_{0}+\frac{K_{0}}{\alpha_{0}}$ where $K_{0}=2,000,000$. This gives $\widehat{K}_{0}=969265$, and Black-Scholes parameters

$y=0.02, r=0.08, \tau=0.25, x=1,000,000, K=969,265, d_{1}=0.512170, d_{2}=0.412170$

The value of the option to delay the commencement of the project is $\$ 775,385.63$. The expected gold price in three months time is $\$ 1,015.113 / \mathrm{oz}$, when the expected value of the project's future cashflows is $1015113 \times 11.870898-900000 \times 10.562276=\$ 2,544,254.91$. This includes a range of outcomes both positive and negative. The expected NPV of the project in three months is $\$ 544,254.91$. The project value today is $\$ 533,477.94$. The right (not obligation) to commence the project in three months for a $\$ 2 \mathrm{~m}$ outlay is worth $\$ 775,385.63$.

Example 3: Suppose one year after project commencement we have the right to abandon and recover the project's salvage value, at which time the project has two years of remaining life. We can value this right to abandon at the date when the project commences. Assuming the project has salvage value $\$ 1,000,000$, we compute the parameters for the valuation of the put option:

The put option multiple is $\alpha_{m}=A(q, 0, n-m)=7.910634$, the put option exercise price is

$$
\widehat{K}_{m}=\frac{S_{m}}{\alpha_{m}}+\beta_{m}=\frac{1000000}{7.910634}+832703.91=959116.03
$$

where $\beta_{m}=C \frac{A(r, m+1, n)}{A(q, m+1, n)}=900000 \frac{7.319129}{7.910634}=832703.91$. The value of the right to abandon equals 7.910634 put options over $1000 \mathrm{oz}$ of gold with exercise price 959116 and a term of one year. If the price of gold at the time the project commences is $\$ 1,015.11 / \mathrm{oz}$ then the value of the abandonment put option follows with the following Black-Scholes parameters:

$$
\begin{aligned}
y=0.02, r=0.08, \tau & =1.0, x=1015113, K=959116, d_{1}=0.683716, d_{2}=0.483716 \\
P & =K e^{-r \tau} N\left(-d_{2}\right)-x e^{-y \tau} N\left(-d_{1}\right)=32,423.06
\end{aligned}
$$

The value of the abandonment put option is $7.91063432,423=256,487$. The time $T_{0}$ value (at project commencement) of the project's future cashflows including the value of the right to abandon is computed as follows: the present value of the salvage value obtained by abandoning the mine project $(\$ 923,116.35)$ plus the present value of the mine's cashflows during the first year of operation $(\$ 609,887.24)$ plus the value of 7.910634 call options over gold with the same parameters as for the put option above $(\$ 1,123,783.55)$, which sum to $\$ 2,656,787.13$. This is the value of the mine project's future cashflows allowing for the right to abandon one year after the project commences. The value of the project's future cashflows at the time of commencement without the abandonment option is $\$ 2,544,254.91$. The right to abandon the project after one year increases this value by $\$ 112,532.23$ and increases the NPV to $\$ 656,787.13$

Example 4: Suppose one year after project commencement we have the right to expand by opening up a new mine with a different life and cost of extraction. The new project has a four year life once commenced and cost of extraction $\$ 1000 / \mathrm{oz}$. Assume it costs $\$ 3 \mathrm{~m}$ to initiate the project. Using the same parameters as above, the value of the option to expand the project in one year's time is equal to $\hat{\alpha}_{p}=15.831163$ call options over gold with an exercise price $\widehat{K}_{p}=1045790$, a term of one year and a spot price of 1015113 .

Since $y=0.02, r=0.08, \tau=1.0, x=1015113, K=1045790, d_{1}=0.251135, d_{2}=0.051135, c=93778.53$, the value of the expansion option is therefore $\$ 1,484,623.14$

Example 5: We numerically compute the critical gold price for which we would proceed with a flexible project which includes the rights to abandon and to expand at future times $T_{m}$ and $T_{p}$, for a $\$ 2 \mathrm{~m}$ outlay. The value of the cash-flows at time $T_{0}<T_{m}, T_{p}$ is :

$$
V_{T_{0}}=\alpha_{0}\left(X_{T_{0}}-\beta_{0}\right)+S_{m} e^{-r\left(T_{m}-T_{0}\right)}+\alpha_{m} C\left(X_{T_{0}}, \widehat{K}_{m}, T_{m}-T_{0}\right)+\hat{\alpha}_{p} C\left(X_{T_{0}}, \widehat{K}_{p}, T_{p}-T_{0}\right)
$$

Now $\quad \alpha_{0}=3.975093, \quad \beta_{0}=861,685.92, \quad \hat{\alpha}_{p}=7.910634, \widehat{K}_{p}=1304462.92, \quad \alpha_{m}=7.910634 \widehat{K}_{m}=$ $959,116.03$ and $S_{m} e^{-r\left(T_{m}-T_{0}\right)}=923116.35$. Excel's goal-seek tool computes the critical gold price for which $V_{T_{0}}$ equals $\$ 2 \mathrm{~m}$ to be $\$ 881.79 / \mathrm{oz}$. 
Example 6: We now compute the project value in Example 5 with a $T_{0}=3$ month option to delay before investing the $\$ 2 \mathrm{~m}$ to initiate, and with the further options to either abandon or expand. Assume an initial gold price of $\$ 1000$ /oz. The time $t<T_{0}$ values of the components in expression (3) are as follows.

First, an asset binary option with value $x e^{-q \tau_{0}} N\left(d_{a}\left(x, \tau_{0}\right)\right)=922949$ with the same parameters as in Example 5, and with $x=\$ 1 \mathrm{~m}$.

Second, a first order bond binary option $e^{-r \tau_{0}}\left(S_{m} e^{-r\left(T_{m}-T_{0}\right)}-K_{0}-\alpha_{0} \beta_{0}\right) N\left(d_{a}^{\prime}\left(x, \tau_{0}\right)\right)$ where $d_{a}^{\prime}\left(x, \tau_{0}\right)=$ 1.35797 and $S_{m} e^{-r\left(T_{m}-T_{0}\right)}-K_{0}-\alpha_{0} \beta_{0}=-4,502,170$

Third, a generalized compound option $\alpha_{m} Q_{a, \widehat{K}_{m}}^{++}$with parameters:

$$
\begin{gathered}
\tau_{0}=0.25, \tau_{m}=1.25, \widehat{\alpha}_{m}=7.910634, \widehat{K}_{m}=959116.03 \\
d_{\widehat{K}_{m}}\left(x, \tau_{m}\right)=0.6339, d_{\widehat{K}_{m}}^{\prime}\left(x, \tau_{m}\right)=0.4103, \rho_{m}=0.447214 \\
Q_{a, \widehat{K}_{m}}^{++}=x e^{-q \tau_{2}} N_{2}\left(d_{a}\left(x, \tau_{0}\right), d_{\widehat{K}_{m}}\left(x, \tau_{m}\right) ; \rho_{m}\right)-\widehat{K}_{m} e^{-r \tau_{2}} N_{2}\left(d_{a}^{\prime}\left(x, \tau_{0}\right), d_{\widehat{K}_{m}}^{\prime}\left(x, \tau_{m}\right) ; \rho_{m}\right)=142904
\end{gathered}
$$

Fourth, a generalized compound option $\hat{\alpha}_{p} Q_{a, K_{p}}^{++}$with parameters:

$$
\begin{gathered}
\tau_{0}=0.25, \tau_{p}=1.25, \widehat{\alpha}_{p}=7.910634, \widehat{K}_{p}=1045790 \\
d_{\widehat{K}_{p}}\left(x, \tau_{p}\right)=0.246985, d_{\widehat{K}_{p}}^{\prime}\left(x, \tau_{p}\right)=0.0233777, \rho_{p}=0.447214 \\
Q_{a, \widehat{K}_{p}}^{++}=x e^{-q \tau_{2}} N_{2}\left(d_{a}\left(x, \tau_{0}\right), d_{\widehat{K}_{p}}\left(x, \tau_{p}\right) ; \rho_{p}\right)-\widehat{K}_{p} e^{-r \tau_{2}} N_{2}\left(d_{a}^{\prime}\left(x, \tau_{0}\right), d_{\widehat{K}_{p}}^{\prime}\left(x, \tau_{p}\right) ; \rho_{p}\right)=98847.50
\end{gathered}
$$

The value of the project with an additional three month option to defer commencement before initiation therefore comes to $\$ 2,336,100$.

\section{Conclusion}

Mining projects involve the extraction and processing of commodities where the commodity price determines a venture's ultimate feasibility. In most real-world projects, operators may have several flexibilities available such as the option to defer commencement of mining operations, to expand mining operations and/or to abandon mining operations at some future time. Through examples motivated by a hypothetical gold mining venture, we have presented new analytic formulae for the evaluation of mining projects with the above flexibilities as non-standard compound options over the underlying commodity. We also discussed the extension to project versions with the further flexibility of project cancellation when the underlying commodity price falls below some threshold level of viability before project initiation, and presented analytical valuation formulae with knock-out barrier option features. Several example numerical implementations of our formulae using Excel/VBA were also presented. Option valuation is traditionally accomplished by numerical methods, although we presented closed-form formulae for our examples. Extensions to projects with knock-in barrier features are also possible, expressible in terms similar to the results presented here. Such versions model the situation where the commodity price needs to rise to meet some threshold of profitability for operations to be viable. More complicated extensions allowing for greater degrees of flexibility are also possible, but with increased complexity in the valuation formulae, which we leave for subsequent publications.

\section{References}

Arnold, T. and Shockley, R.L. (2002). Real options analysis and the assumptions of the NPV rule. [Online] Available: http://www.realoptions.org

Black, F. and Scholes, M. (1973). The pricing of options and corporate liabilities. Journal of political economy, 81(3), 637-654. http://dx.doi.org/10.1086/260062

Boyle, P. (1977). Options: A monte-carlo approach. Journal of Financial Economics, 4(3), 323-338. http://dx.doi.org/10.1016/0304-405X(77)90005-8

Buchen, P. (2001). Image options and the road to barriers. Risk Magazine, 14(9), 127-130.

Buchen, P. (2004). The pricing of dual-expiry exotics. Quantitative Finance, 4(1), 101-108. http://dx.doi.org/10.1088/1469-7688/4/1/009

Buchen, P. and Konstandatos, O. (2009). A new approach to pricing double-barrier options with arbitrary payoffs and exponential boundaries. Applied Mathematical. Finance, 16(6), 497-515. http://dx.doi.org/10.1080/13504860903075480

Buchen, P., Konstandatos, O., and Kyng, T. (2009). Images and barriers on the road to real options valuation. Proceedings of the 18th IMACS World Congress MODSIM2009, 1486-1492.

Cox, J., S. Ross, and M. Rubinstein, (1979). Option pricing: a simplified approach, Journal of Financial Economics, 7, 44-50. http://dx.doi.org/10.1016/0304-405X(79)90015-1 
Dixit, A., Pindyck, R., and Davis, G. (1994). Investment under uncertainty. Princeton University Press Princeton, NJ. Geske, R. (1979). The valuation of compound options, Journal of Financial Economics, 63-81. http://dx.doi.org/10.1016/0304-405X(79)90022-9

Guj, P. (2011). A practical real option methodology for the evaluation of farm-in/out joint venture agreements in mineral exploration. Resources Policy 36(2011)80-90. http://dx.doi.org/10.1016/j.resourpol.2010.08.006

Harrison (1985). Brownian motion and stochastic flow systems. John Wiley and Sons, New York.

Harrison, J. and Pliska, S. (1981) Martingales and stochastic integrals in the theory of continuous trading. Stochastic Processes and their Applications, 11(3), 215-260. http://dx.doi.org/10.1016/0304-4149(81)90026-0

Hemantha S.B. Herath \& Chan S. Park. (2002). Multi-Stage Capital Investment Opportunities as Compound Real Options, The Engineering Economist: A Journal Devoted to the Problems of Capital Investment, 47:1, 1-27.

Hull, J. (2009). Options, futures, and other derivatives. Pearson Education India.

Hull, J. and White A. (1990). Valuing Derivative Securities Using the Explicit Finite Difference Method, Journal of Financial and Quantitative Analysis, 25(1), 87-100. http://dx.doi.org/10.2307/2330889

Kac, M. (1949). On distributions of certain Wiener functionals. Transactions of the American Mathematical Society, 65 (1), 1-13. http://dx.doi.org/10.1090/S0002-9947-1949-0027960-X

Konstandatos, O. (2003). A new framework for pricing barrier and lookback options. PhD Thesis, The University of Sydney, Sydney Australia.

Konstandatos, O. (2008). Pricing path dependent options: a comprehensive mathematical framework. VDM Verlag, Saarbrucken, Germany

Kyng, T. (2011). Application of Black-Scholes exotic option pricing theory to real options and executive share options. PhD Thesis, The University of Sydney, Sydney Australia.

Lee, M., Yeh, F. and Chen, P. (2008). The generalised sequential compound options pricing and sensitivity analysis. Mathematical Social Sciences, 55 (2008), 38-54. http://dx.doi.org/10.1016/j.mathsocsci.2007.07.001

Merton, R. (1973). Theory of rational option pricing. Bell Journal of Economics and Management Science, 4(1), 141-183. http://dx.doi.org/10.2307/3003143

Merton, R. (1998). Applications of option pricing theory: Twenty-five years later. American Economic Review, 88(6), 323-349.

Myers, S. (1977). Determinants of capital borrowing. Journal of Financial Economics, 5.

Pendharkar, C.P. (2010). Valuing inter-dependent multi-stage IT investments: A real options approach, European Journal of Operational Research, 201847-859

Rich, D. (1994). The mathematical foundations of barrier option pricing theory, Advances in Futures and Options Research, 7, 267-311.

Rubinstein, M. and Reiner, E. (1991). Breaking down the barriers. Risk, 4(8), p 28-35.

Samuelson, P. (1965). Rational theory of warrant pricing. Industrial Management Rev, 6, 3-31.

Topal. E. (2008). Evaluation of a mining project using Discounted Cash Flow analysis, Decision Tree analysis, Monte Carlo Simulation and Real Options using an example. International Journal of Mining and Mineral Engineering, Vol. 1, No. 1, 2008

Trigeorgis, L. (1993). The nature of option interactions and the valuation of investments with multiple real options. Journal of Financial and Quantitative Analysis, 28(1), 1-20. http://dx.doi.org/10.2307/2331148

Wilmott, P., Howison, S. and Dewynne, J. (1995). The mathematics of financial derivatives: a student introduction. Cambridge University Press. http://dx.doi.org/10.1017/CBO9780511812545 